\title{
Front Matter: Volume 9510
}

, "Front Matter: Volume 9510," Proc. SPIE 9510, EUV and X-ray Optics: Synergy between Laboratory and Space IV, 951001 (4 June 2015); doi: 10.1117/12.2199420

SPIE. Event: SPIE Optics + Optoelectronics, 2015, Prague, Czech Republic 


\section{PROCEEDINGS OF SPIE}

\section{EUV and X-ray Optics: Synergy between Laboratory and Space IV}

René Hudec

Ladislav Pina

Editors

13-14 April 2015

Prague, Czech Republic

Sponsored by

SPIE

Cooperating Organizations

HiPER Project (United Kingdom)

ELI Beamlines (Czech Republic)

Laserlab Europe

Published By

SPIE 
The papers included in this volume were part of the technical conference cited on the cover and title page. Papers were selected and subject to review by the editors and conference program committee. Some conference presentations may not be available for publication. The papers published in these proceedings reflect the work and thoughts of the authors and are published herein as submitted. The publisher is not responsible for the validity of the information or for any outcomes resulting from reliance thereon.

Please use the following format to cite material from this book:

Author(s), "Title of Paper," in EUV and X-ray Optics: Synergy between Laboratory and Space IV, edited by René Hudec, Ladislav Pina, Proceedings of SPIE Vol. 9510 (SPIE, Bellingham, WA, 2015) Article CID Number.

ISSN: 0277-786X

ISBN: 9781628416312

Published by

SPIE

P.O. Box 10, Bellingham, Washington 98227-0010 USA

Telephone +1 3606763290 (Pacific Time) · Fax +1 3606471445

SPIE.org

Copyright (C) 2015, Society of Photo-Optical Instrumentation Engineers.

Copying of material in this book for internal or personal use, or for the internal or personal use of specific clients, beyond the fair use provisions granted by the U.S. Copyright Law is authorized by SPIE subject to payment of copying fees. The Transactional Reporting Service base fee for this volume is $\$ 18.00$ per article (or portion thereof), which should be paid directly to the Copyright Clearance Center (CCC), 222 Rosewood Drive, Danvers, MA 01923. Payment may also be made electronically through CCC Online at copyright.com. Other copying for republication, resale, advertising or promotion, or any form of systematic or multiple reproduction of any material in this book is prohibited except with permission in writing from the publisher. The CCC fee code is 0277-786X/15/\$18.00.

Printed in the United States of America.

Publication of record for individual papers is online in the SPIE Digital Library.

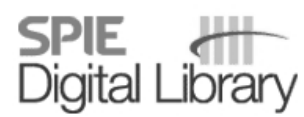

SPIEDigitallibrary.org

Paper Numbering: Proceedings of SPIE follow an e-First publication model, with papers published first online and then in print. Papers are published as they are submitted and meet publication criteria. A unique citation identifier (CID) number is assigned to each article at the time of the first publication. Utilization of CIDs allows articles to be fully citable as soon as they are published online, and connects the same identifier to all online, print, and electronic versions of the publication. SPIE uses a six-digit CID article numbering system in which:

- The first four digits correspond to the SPIE volume number.

- The last two digits indicate publication order within the volume using a Base 36 numbering

system employing both numerals and letters. These two-number sets start with 00, 01, 02, 03, 04, $05,06,07,08,09,0 \mathrm{~A}, 0 \mathrm{~B} \ldots \mathrm{OZ}$, followed by 10-1Z, 20-2Z, etc.

The CID Number appears on each page of the manuscript. The complete citation is used on the first page, and an abbreviated version on subsequent pages. 


\title{
Contents
}

\author{
vii Authors \\ ix Conference Committee
}

\section{ASTRONOMICAL X-RAY OPTICS I}

951002 Beyond Chandra: the x-ray Surveyor (Invited Paper) [9510-1]

$951003 \quad$ X-ray optics at NASA Marshall Space Flight Center [9510-2]

951004 Alternative technologies and arrangements for future space $x$-ray telescopes [9510-3]

\section{ASTRONOMICAL X-RAY OPTICS II}

$951005 \quad$ X-ray monitoring for astrophysical applications on Cubesat [9510-5]

951006 X-ray characterization of curved crystals for hard x-ray astronomy [9510-6]

951007 ISS-Lobster: a low-cost wide-field x-ray transient detector on the ISS [9510-7]

951008 Polarizers for a spectral range centered at $121.6 \mathrm{~nm}$ operating by reflectance or by transmittance [9510-8]

951009 Transverse $x$-ray scattering on random rough surfaces [9510-9]

\section{ASTRONOMICAL X-RAY OPTICS III}

9510 0A Applications of lobster eye optics [9510-10]

$9510 \mathrm{OB} \quad \mathrm{He}+$ ions damage on optical coatings for solar missions [9510-11]

$95100 \mathrm{C}$ Analytical description of lobster eye and similar multi-foil optics [9510-12]

9510 OD Preliminary design of optics for nano-satellite monitor [9510-13]

9510 OE Achieving zero stress in iridium, chromium, and nickel thin films [9510-28]

\section{DIFFRACTIVE AND REFRACTIVE X-RAY OPTICS}

$95100 \mathrm{G}$ Self standing curved crystals for gamma ray focusing [9510-16]

$9510 \mathrm{OH}$ Dynamical diffraction approach of deformed crystals using finite element method [9510-17] 
$95100 \mathrm{~J}$ Narrowband filters for the FUV range [9510-19]

$9510 \mathrm{OL} \quad$ Low-stress coatings for sputtered-sliced Fresnel zone plates and multilayer Lave lenses [9510-21]

\section{X-RAY MICROSCOPES AND ACTIVE X-RAY OPTICS}

$95100 \mathrm{M}$ A compact "water-window" microscope with 60-nm spatial resolution based on a double stream gas-puff target and Fresnel zone plate optics [9510-22]

9510 ON Nanoscale imaging and optimization of a compact "water window" SXR microscope [9510-23]

951000 Performance testing of a novel off-plane reflection grating and silicon pore optic spectrograph at PANTER [9510-37]

\section{COHERENT RADIATION/LASERS}

9510 OP Photoionized plasmas in laboratory: a connection to astrophysics and planetary sciences [9510-24]

$95100 Q \quad$ Laser plasma sources of soft $x$-rays and extreme ultraviolet (EUV) for application in science and technology [9510-25]

\section{MULTILAYER X-RAY OPTICS}

9510 OS Diffraction gratings based on asymmetric-cut multilayers [9510-27]

9510 OT Optical and structural characterization of CeO2/B4C multilayers near boron K-edge energy [9510-29]

$95100 \mathrm{U}$ Overview of the multilayer-Fresnel zone plate and the kinoform lens development at MPI for intelligent systems (Best Student Paper Award) [9510-38]

9510 OV Characterization of nanoscale multilayer structures upon thermal annealing [9510-30]

$95100 \mathrm{X}$ Periodic $\mathrm{Co} / \mathrm{C}, \mathrm{Cr} / \mathrm{C}$, and $\mathrm{CoCr} / \mathrm{C}$ soft $\mathrm{x}$-ray multilayers prepared by $\mathrm{N}$ reactive sputtering [9510-32]

9510 OY Development of XUV multilayer gratings with high resolution and high efficiency [9510-33]

\section{POSTER SESSION}

$95100 Z$ Characterization of $\mathrm{TiO}_{2}$ thin films in the EUV and soft X-ray region [9510-34]

951010 Radiometric modelling of a space optical instrument: an example of application to PHEBUS [9510-35] 
951011 EUV ablation: a study of the process [9510-36]

951013 Time-resolved EUV spectra from nitrogen Z-pinching capillary discharge [9510-40]

951014 Focusing and photon flux measurements of the 2.88-nm radiation at the sample plane of the soft $x$-ray microscope, based on capillary discharge source [9510-41] 
Proc. of SPIE Vol. $9510951001-6$

Downloaded From: https://www.spiedigitallibrary.org/conference-proceedings-of-spie on 26 Apr 2023 Terms of Use: https://www.spiedigitallibrary.org/terms-of-use 


\section{Authors}

Numbers in the index correspond to the last two digits of the six-digit citation identifier (CID) article numbering system used in Proceedings of SPIE. The first four digits reflect the volume number. Base 36 numbering is employed for the last two digits and indicates the order of articles within the volume. Numbers start with 00, 01, 02, 03, 04, 05, 06, 07, 08, 09, OA, OB...0Z, followed by 10-12, 20-2Z, etc.

Ackermann, Marcelo, 00

Adjei, Daniel, $0 M, 0 Q$

Ahad, Inam Ul, OQ, 11

Allured, Ryan, 00

Ames, A., OE

Atkins, Carolyn, 03

Ayele, Mesfin Getachew, $O Q$

Aznárez, José A., 08, 0J

Bacco, Davide, OB, 10

Bajt, Saša, OS

Barbera, Marco, OC

Barthelmy, Scott, 07

Bartnik, Andrzej, OM, ON, OP, OQ, 11

Belas, E., 05

Bijkerk, F., OV

Bonnini, Elisa, 06, 0G

Böttger, R., OB

Braun, Stefan, OL

Brigo, L., $\mathrm{OZ}$

Broadway, David M., 03, OE

Brose, S., OT

Bruni, R., OE

Buffagni, Elisa, 06, OG

Burian, Tomáš, 11

Burwitz, Vadim, 00

Calvillo, L., OZ

Camp, Jordan, 07

Capobianco, Gerardo, 08

Cash, W., 05

Cerna, D., 05

Chapman, Henry N., OS

Chodukowski, T., OP

Cikhardt, J., OP

Cikhardtova, B., OP

Comisso, A., OT, OZ

Corso, Alain Jody, OB, 10

Cui, Mingqi, $O X$

Dániel, V., 05

DeRoo, Casey, 00

Donovan, Benjamin D., 00

Dostal, J., OP

Doyle, S., OG

Dudzak, R., OP

Elsner, Ronald F., 03

Endo, Akira, 11

Ferrari, Claudio, 06, 0G

Ferrero, $\mathrm{C} ., \mathrm{OH}$

Fiedorowicz, Henryk, OM, ON, OP, OQ

Fineschi, Silvano, 08
Fok, Tomasz, OM, OP, OQ

Frassetto, Fabio, 08

Frontera, Filippo, 06

Fu, Liping, OJ

Gaskin, Jessica A., 02, 03

Gawlitza, Peter, OL

Gehrels, Neil, 07

Gerlin, Francesca, OB, OZ, 10

Giglia, Angelo, 08, OT, OZ

Granozzi, G., OZ

Grévent, Corinne, OU

Gubarev, Mikhail V., 03, OE

Guigay, J.-P., $\mathrm{OH}$

Günther, Ramses, 00

Gurgew, Danielle, OE

Hájková, Věra, 11

Hartner, Gisela D., 00

Hrebícek, J., OP

Huang, Qiushi, OX, OY

Hudec, René, 04, 05, OA, OC, OD

Inneman, A., 04, 05, 0A

Jakubek, J., 05

Jančárek, Alexandr, OM, 13, 14

Jarocki, Roman, ON, OP, OQ

Jiang, Li, OX

Juha, Libor, 11

Juschkin, L., OT

Kalinowska, Z., OP

Keskinbora, Kahraman, OU

Kilaru, Kiranmayee, 03

Klir, D., OP

Kolodziejczak, Jeffery J., 03

Kostecki, Jerzy, OM, ON, OQ

Kozhevnikov, Igor $V_{\text {., }} \mathrm{OY}$

Krousky, E., OP

Kubec, Adam, OL

Larruquert, Juan I., 08, 0J

Leson, Andreas, $\mathrm{OL}$

Liberatore, Chiara, 11

Limpouch, Jiri, 14

Lis, Tomasz, OE

Makhotkin, I. A., OV

Malvezzi, A. Marco, 08

Mariscal, Jean Francois, 10

Marlowe, Hannah, 00

Marshall, Francis, 07

Massone, Giuseppe, 08

Matulková, Irena, 11

McEntaffer, Randall L., 00 
Medrik, T., OP

Méndez, José A., 08, 0J

Menz, Benedikt, 00

Menzel, Maik, OL

Mikulickova, L., 05

Miles, Drew M., 00

Miotti, Paolo, 08

Mocek, Tomas, 11

Mocella, V., $\mathrm{OH}$

Nannarone, Stefano, 08

Napolitani, E., OB

Nardello, Marco, OB, 0Z, 10

Nawaz, Muhammad Fahad, OM, 0N, 13, 14

Nevrkla, Michal, 13, 14

Nicolosi, Piergiorgio, OT, OZ, 10

O'Dell, Stephen L., 03, OE

Parkman, Tomáš, 13

Pavlica, R., 05

Pelizzo, Maria Guglielmina, OB, 10

Petre, Rob, 07

Pfeifer, M., OP

Pina, Ladislav, 04, 05, 0A, OP, 11, 14

Pisarczyk, T., OP

Polak, J., 05

Prasciolu, Mauro, OS

Ptak, Andy, 07

Quémerais, Eric, 10

Racusin, Judith, 07

Ramsey, Brian D., 03, OE

Rezac, K., OP

Roche, Jacqueline M., 03

Rodríguez-de Marcos, Luis, 08, 0J

Rouanet, Nicolas, 10

Schütz, Gisela, OU

Sertsu, M. G., OT, OZ

Sieger, L., 05

Šimon, Vojtěch, OD

Skala, J., OP

Smith, Randall K., 00

Swartz, Douglas A., 03

Szczurek, Anna, $0 Q$

Szczurek, Mirosław, ON, OP, OQ

Tananbaum, Harvey, 02

Tennant, Allyn F., 03

Tessarolo, Enrico, OB, OZ, 10

Tichý, Vladimír, OA, OC, OD

Torrisi, Alfio, OM, ON, OQ

Toufarová, Martina, 11

Tunca Sanli, Umut, OU

Turňová, Jana, OM

Tutt, James H., $0 \mathrm{O}$

Ullschmied, J., OP

Vacanti, Giuseppe, 00

van de Kruijs, R. W. E., OV

Vikhlinin, Alexey, 02

Virgilli, Enrico, 06

Vondrová, Šárka, OM

Vrbová, Miroslava, 13

Vyšín, Luděk, 11
Wachulak, Przemyslaw, OM, ON, OP, OQ, 14

Wang, Hongchang, OX

Wang, Zhanshan S., OT, OX, OY

Węgrzyński, Łukasz, OM, ON, OP, OQ

Weimer, Jeffrey, OE

Weisskopf, Martin C., 02, 03

Wen, Mingwu, OX

Wu, Yanaing, OY

Yakshin, A., OV

Yang, Xiaowei, OY

Yanson, Alex, 00

Yi, Rongqing, $\mathrm{OX}$

Zameshin, A., OV

Zavlin, Vyacheslav E., 03

Zhang, Zhong, OX

Zhao, Jun, OY

Zhao, Ping, 09

Zuccon, Sara, OB, 10

Zuppella, Paola, OB, 10

viii

Proc. of SPIE Vol. $9510951001-8$ 


\title{
Conference Committee
}

\author{
Symposium Chairs
}

Jiri Homola, Institute of Photonics and Electronics of the ASCR, v.v.i.

(Czech Republic)

Chris Edwards, Central Laser Facility, Science and Technology

Facilities Council (United Kingdom)

Mike Dunne, SLAC National Accelerator Laboratory (United States)

and Linac Coherent Light Source (United States)

Ivo Rendina, Istituto per la Microelettronica e Microsistemi (Italy)

Honorary Symposium Chair

Miroslav Miller, Institute of Photonics and Electronics of the ASCR, v.v.i

(Czech Republic)

Conference Chairs

René Hudec, Astronomical Institute of the ASCR, v.v.i.

(Czech Republic) and Czech Technical University in Prague

(Czech Republic)

Ladislav Pina, Czech Technical University in Prague (Czech Republic)

Conference Programme Committee

Webster Cash, University of Colorado at Boulder (United States)

Henryk Fiedorowicz, Military University of Technology (Poland)

Ali M. Khounsary, X-ray Optics, Inc. (United States)

Randall L. McEntaffer, The University of lowa (United States)

Stephen L. O'Dell, NASA Marshall Space Flight Center (United States)

Giovanni Pareschi, INAF - Osservatorio Astronomico di Brera (Italy)

Yuriy Ya Platonov, Rigaku Innovative Technologies, Inc. (United States)

Paul B. Reid, Harvard-Smithsonian Center for Astrophysics (United States)

Bedřich Rus, ELI Beamlines (Czech Republic) and Institute of Physics of the ASCR, v.v.i. (Czech Republic)

Anatoly Snigirev, ESRF - The European Synchrotron (France)

Peter Z. Takacs, Brookhaven National Laboratory (United States)

Melville P. Ulmer, Northwestern University (United States)

David L. Windt, Reflective X-Ray Optics LLC (United States)

William W. Zhang, NASA Goddard Space Flight Center (United States) 
Session Chairs

1 Astronomical X-ray Optics I

Jordan Camp, NASA Goddard Space Flight Center (United States)

2 Astronomical X-ray Optics II

Martin C. Weisskopf, NASA Marshall Space Flight Center (United States)

3 Astronomical X-ray Optics III

Stefan Braun, Fraunhofer IWS Dresden (Germany)

4 Various and Integrated Devices and Systems

Stefan Braun, Fraunhofer IWS Dresden (Germany)

5 Diffractive and Refractive X-ray Optics

Stephen L. O'Dell, NASA Marshall Space Flight Center (United States)

6 X-ray Microscopes and Active X-ray Optics

Igor A. Makhotkin, Universiteit Twente (Netherlands)

7 Coherent Radiation/Lasers

Igor A. Makhotkin, Universiteit Twente (Netherlands)

8 Multilayer X-ray Optics

Hannah R. Marlowe, The University of lowa (United States) 